\title{
Analog Frontend for Multichannel Neuronal Recording System \\ with Spike and LFP Separation
}

\author{
Yevgeny Perelman and Ran Ginosar, \\ VLSI Systems Research Center, Department of Electrical Engineering, \\ Technion-Israel Institute of Technology, Haifa 32000, Israel \\ perelman@tx.technion.ac.il, ran@ee.technion.ac.il
}

\section{INTRODUCTION}

Recent advances in fabrication of MEMS microelectrode arrays [1], [2], together with the ability of coupling the arrays directly to VLSI chips, allow simultaneous monitoring of tens and even hundreds of neurons. Moreover, clinical applications of brain-machine interfaces may require monitoring of much larger populations, even hundreds and thousands of neurons [3].

With this large a number of recording units, communicating raw neuronal signals results in prohibitively large data rates [4]. When sampled with 20Ksps, eight bit precision, even a hundred of electrodes would generate $16 \mathrm{Mbps}$, too large for common methods of low-power wireless communications. Evidently, some form of data reduction must be applied prior to communication.

It is possible to detect the presence of neuronal spikes as demonstrated in [4] and communicate only active portions of recorded signals. Assuming an electrode might "sense" two or three units which fire 20 times per second on average, and taking the firing event length to be $2 \mathrm{mSec}$, only ten times data rate reduction can be achieved. Further reduction can be provided by restricting the communicated information to mere indications of spike presence. 
An extracellular microelectrode typically senses activity from several units adjacent to its tip. Spike sorting [5] applies classification techniques to assign spike waveforms of different shapes to different units. With on-chip spike sorting, the data bandwidth is reduced to 200Kbps (almost a hundred times) for the figures above, assuming a 32bit message generated for every spike.

Another reason for on-chip sorting is brought in [6]. In autonomous motor prosthetics, assuming that every spike coming from a certain electrode is generated by the same unit might prove not sufficiently accurate enough for movement trajectory calculations. [6] shows also that implementation of existing algorithms for on chip spike sorting is feasible in terms of power dissipation.

\section{Motivation of this work}

In a signal recorded by an extracellular microelectrode, neuronal firing activity occupies the $100-10.000 \mathrm{~Hz}$ frequency band; its amplitude is typically lower than $500 \mu \mathrm{V}$. The Local Field Potential (LFP) occupies the lower frequencies, below $100 \mathrm{~Hz}$, with amplitudes below $5 \mathrm{mV}$. The signal-to-noise ratio of the combined signal is rather large: as the microelectrode noise [7] and background noise of cortical activity [8] are typically $5 \mu \mathrm{V}$, it may reach $60 \mathrm{~dB}$.

Since the LFP must be filtered out prior to spike sorting, it is possible to block it right at the front-end [9], by high-pass filtering below $100 \mathrm{~Hz}$. It was shown, however, that LFP carries important information [10], [11]. Several front-end circuits pass the LFP band intact: [12], [13], [14]. They block the large input DC offsets, typical for neuronal signals, by high-pass filtering below $1 \mathrm{~Hz}$. As the entire combined signal is passed, the minimal required precision of subsequent data acquisition is 10 bit, defined by the signal SNR. The maximal gain is limited by the LFP magnitude and chip supply voltage. Since the firing activity (SPK) has ten timer lower magnitude than the LFP, it can be amplified only to one tenth of the output swing.

To overcome these limitations, we suggest splitting the signal into two bands after the first amplification stage. Thus, the LFP and SPK parts can be processed by separate channels, amplifying both to the full swing. Consequently, the system dynamic ange needs only be a 100 , as determined by the SNR of the SPK signal. Thus, no more than seven bits data acquisition is required.

In this paper we present an integrated CMOS front-end for neuronal recordings from implanted electrodes capable of delivering both spike data and the local field potential from twelve truedifferential recording channels. The front-end is intended for further integration on a head-stage with an external wireless interface.

Following the overall system architecture along with some basic parameter constraints and the preamp circuit, the paper presents the measurement results of the fabricated test chip. 


\section{ARCHITECTURE}

A $0.35 \mu \mathrm{m}$ CMOS twelve-channel neuronal recording front-end IC was designed with a truedifferential inputs, so that each electrode can be referenced to any point in the system. The amplification channel was designed to have an RMS noise level of $2-3 \mu \mathrm{V}$ in the band of $0.2-$ $10 \mathrm{kHz}$.

The block diagram of a single channel is shown in Fig. 1.

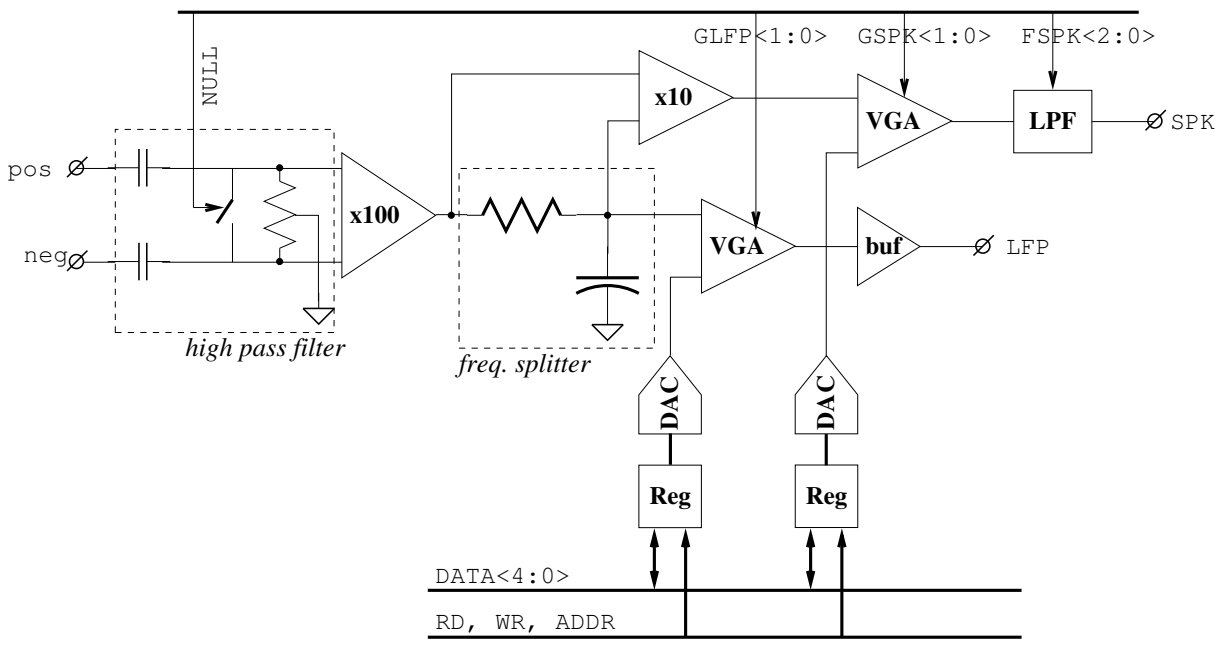

Fig. 1. A single channel block diagram

The input signal is first cleared of the DC offsets by a single-pole input high-pass filter. The corner frequency should be of several Hertz, in order to pass the LFP part of the signal intact. The resistors $(8 \mathrm{M} \Omega)$ were placed on-chip, whereas the capacitors must be added extrnally. As the IC was intended to couple to $0.1-1 \mathrm{M} \Omega$ electrodes, $8 \mathrm{M} \Omega$ input resistance should not present too much of a load.

The first stage provides an amplification of $40 \mathrm{~dB}$ to a single-ended output, which is band-split by a first-order RC filter into high frequency SPK (neuronal firing activity) and low frequency LFP parts. The splitter pole was roughly placed at $200 \mathrm{~Hz}$, by using a $5 \mathrm{M} \Omega$ resistor (high-resistive poly) and 160pF (gate-oxide) capacitor.

The minimal gain to be provided by the first stage is determined by noise constraints as follows. RMS noise introduced by the resistor into the signal (at room temperature) is:

$$
\sqrt{4 k T R f_{0}}=28 \mu V
$$

assuming the output LPF has a steep roll-off above $f_{0}=10 \mathrm{kHz}$. The first stage gain is required to be well above $20 \mathrm{~dB}$ in order to keep the input referred noise of the high splitter resistance below $3 \mu \mathrm{V}$. First stage gain of $40 \mathrm{~dB}$ was taken as a design goal. 
The low frequency LFP signal is amplified by a variable-gain amplifier (VGA) and buffered to chip outputs. The VGA provides digitally selectable gains of 2.5/5/7.5/10x. Thus, the maximum total gain of the LFP channel was designed to be $60 \mathrm{~dB}$.

The high frequency SPK signal is amplified by a factor of ten and by another VGA (to a total maximum of $80 \mathrm{~dB}$ ). Its upper corner frequency is limited by a second-order Bessel LPF, implemented (for the sake of simplicity) as a continuous time Sallen-Key RC biquad [16]. Resistors are implemented as serially-connected polysilicon segments, which can be selectively shortened by means of a three-bit controlling signal. Filter cutoff can be varied that way in the range of $8.5-13 \mathrm{kHz}$.

Although successful implementation of a neuronal signal processing channel based on switched capacitor filter has been reported [9], we have decided to design continuous-time analog circuits, to avoid contaminating the input with switching noise. Switched capacitor implementation for filters can provide for better time-constant accuracy and smaller area, neither of which was our main design goal.

DC offsets of both the SPK and LFP channels have to be compensated: LFP channel amplifies the input preamp offset (hundreds of $\mu \mathrm{V}$, typically) by up to $60 \mathrm{~dB}$; unless compensated, it would limit the dynamic range severely or even saturate the VGA. SPK channel offset is determined by the offset of the $\mathrm{x} 10$ stage amplified by $40 \mathrm{~dB}$, as the DC part of the preamp output signal is cut off by the band splitter. Smaller than LFP, preamp offset is yet significant: the x10 stage has larger input offset compared to the preamp, since the latter uses very large input devices due to the noise requirements.

Offset compensation is carried out by two calibration DACs (one for LFP, one for SPK) applied to the last amplification stages (VGAs). The DACs are implemented as 5-stage R2R resistor ladders, having $400 \mathrm{mV}$ output swing. DAC values are stored in registers that can be individually accessed by the controller FSM through a common bus with five address/data bits and three control bits. The controller calibrates the outputs, one by one, upon de-assertion of the RST signal (Fig. 2).

\section{INPUT PREAMPLIFIER}

The input preamplifier must provide a sufficiently high input impedance in order not to overload the high-impedance recording electrode. The noise level requirement is dictated by the inherent noise of the recording electrode and cortical background noise, both of which having magnitude of several $\mu \mathrm{V}$. In addition, the preamp must have a flat frequency response starting from DC and use no switching circuits. A differential circuit addressing the above requirements is shown on Fig. 3. 


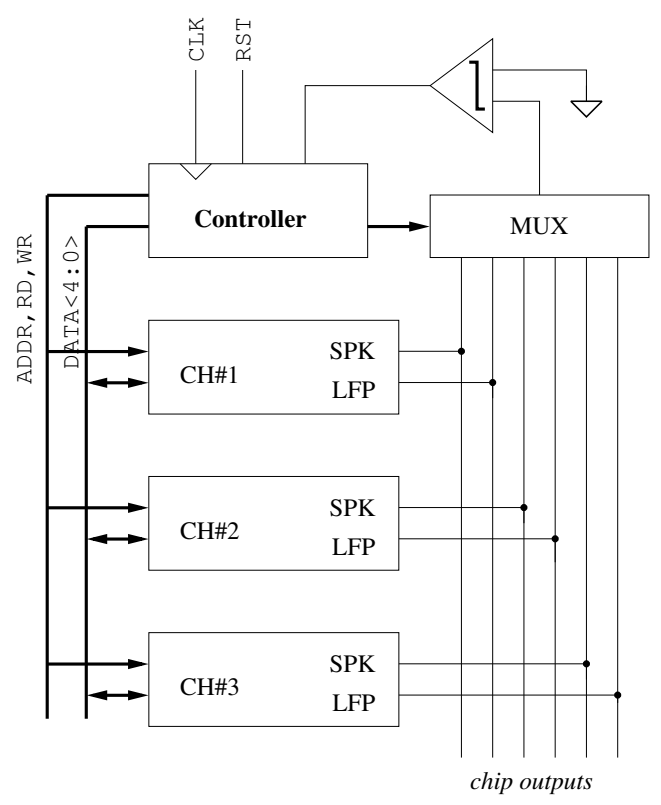

Fig. 2. Chip architecture

This is a degenerated differential cascoded transconductor stage, loaded with a resistor to convert the output current back to voltage. The disadvantage of this circuit is the gain error due to the finite transconductance of input transistors. The gain of the circuit on Fig. 3 is given by:

$$
A=\frac{2 R}{r+r_{m}}=\frac{2 R / r}{1+r_{m} / r}
$$

where $r_{m}$ is the transresistance of $M_{1,2}$. While $r$ can be matched to $R$ by using the same resistor types and employing appropriate layout techniques, there is no straightforward way of matching $r$ to $r_{m}$. The sensitivity to $r_{m}$ can be reduced by reducing the ratio $r_{m} / r$, but there is a limit on how high $r$ can be due to the noise requirements (some $10 \mathrm{k} \Omega$ ) and reducing $r_{m}$ means more power.

Instead, we match $r$ to $r_{m}$ by appropriately controlling the bias currents through $M_{1,2} . M_{1,2}$ are operated in the subthreshold region (the smallest $r_{m}$ for a given $I_{d}$ ) so that $r_{m}$ is inversely proportional to $I_{d}$ :

$$
r_{m}=\frac{\eta V_{t h}}{I_{d}}, \quad V_{t h}=\frac{k T}{q}
$$

$I_{d}$ is given by:

$$
I_{d}=\frac{V_{d d}-V_{g s 3}}{r_{b}}
$$

thus we can write the gain as:

$$
A=\frac{2 R}{r+r_{b} \frac{\eta V_{t h}}{V_{d d}-V_{g s 3}}}
$$




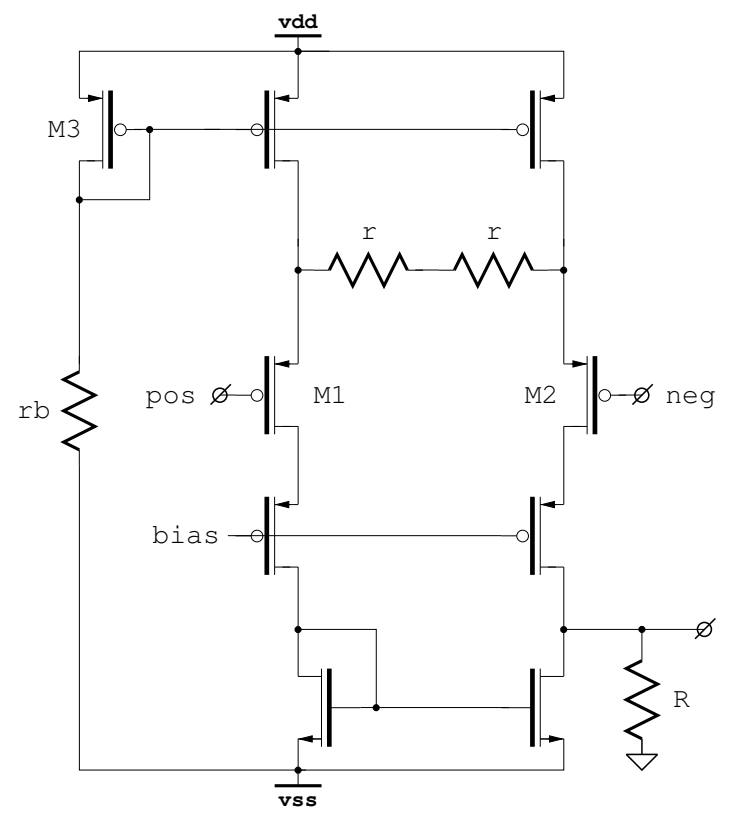

Fig. 3. Input preamplifier circuit topology

We match $r_{b}$ to $r$ and keep $V_{g s 3}$ much lower than $V_{d d}$. Since the ratio of $V_{t h}$ and $V_{d d}$ is small, the above expression becomes weakly dependent on process parameter $\eta$ and on $V_{g s 3}$. The chip is expected to work in constant temperatures (subject body), thus the dependence on $V_{t h}$ is not worrying. One sigma chip-to-chip channel gain variation of less than $2 \%$ was actually measured.

\section{Measurement Results}

The chip was fabricated using AMS $0.35 \mu \mathrm{m}$ quad-metal, double poly CMOS process with 3.3V power supply (Fig. 4).

Ten fabricated chips were tested electrically. Some of the electrical test results are summarized in Tab. I. While most of the design goals were met, note that band splitter frequency is somewhat displaced. This is due to a failure in the band-splitter MOS capacitor biasing circuit.

The measured frequency response (magnitude) for SPK and LFP bands is shown in Fig. 5 (a). The frequency response is quite stable over all the measured chips. Fig. 5 (b) shows input-referred noise power spectral density (PSD) of several channels together with a simulated curve (solid line). $1 / f$ noise dominates over the LFP band and thermal noise dominates the higher frequency SPK band. The increase at the high end is due to out of band components (i.e. above the output LPF cutoff). Power dissipation of $3 \mathrm{~mW}$ per channel was measured. 

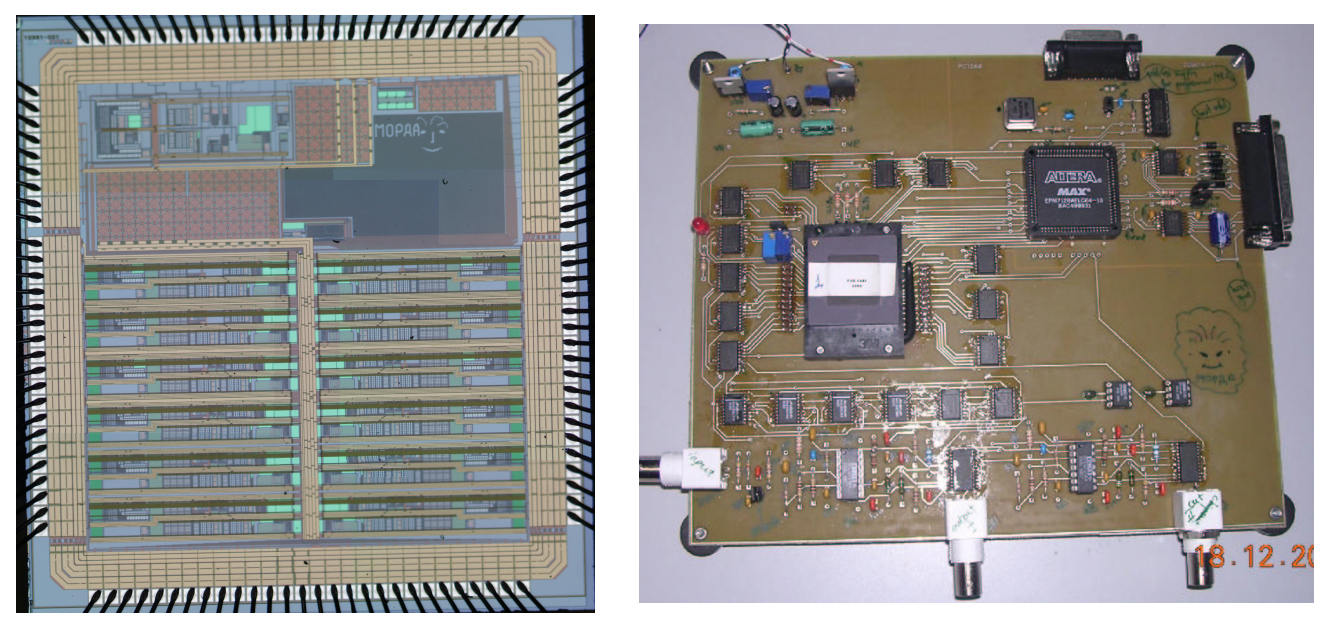

Fig. 4. Chip micrograph and test board

\begin{tabular}{||c|c||}
\hline \hline SPK gain & $77 \mathrm{~dB}$ \\
\hline Output LPF cutoff & $8-12 \mathrm{kHz}$ \\
\hline Band splitter corner & $330 \mathrm{~Hz}$ \\
\hline SPK RMS noise & $3.1 \mu \mathrm{V}$ \\
\hline LFP gain & $58 \mathrm{~dB}$ \\
\hline \multicolumn{2}{|c||}{ TABLE I }
\end{tabular}

ELECTRICAL TEST RESULTS SUMMARY

\section{CONCLUSiOns AND Future Work}

A low noise, dual band, twelve channel differential front-end IC for neuronal recording has been implemented in $0.35 \mu \mathrm{m}$ CMOS technology. The measured signal is separated into LFP (low frequency local field potential) and SPK (high frequency firing activity) bands, spanning $2-200 \mathrm{~Hz}$ and $200 \mathrm{~Hz}-10 \mathrm{kHz}$, respectively. Digitally calibrated offset compensation at both channels and 2-3 stage amplification enable $60 \mathrm{~dB}$ and $80 \mathrm{~dB}$ gains, respectively.

Measurements demonstrate that gain and noise requirements were met. Power consumption was measured at about $3 \mathrm{~mW}$ per channel.

Current research addresses the issues of power dissipation, on-chip integration of the input HPF and A/D conversion. Power dissipation can be reduced by employing active filters (such as $g_{m} / C$ ) and by proper scaling of the later amplification stages to operate on lower currents, as the noise requirements on those stages are relaxed. 


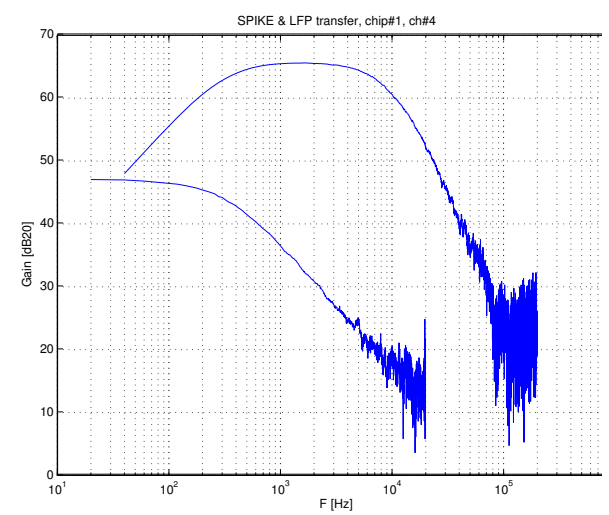

(a) Frequency response

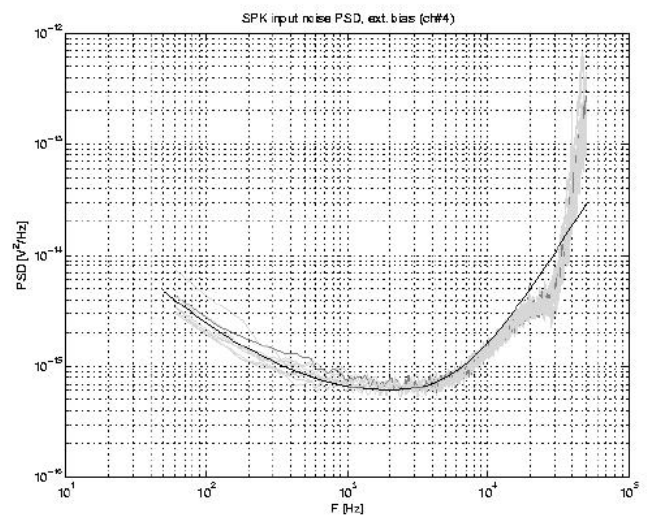

(b) Input noise PSD. Black line simulated PSD.

Fig. 5. Measured channel frequency response and input-referred noise PSD

\section{REFERENCES}

[1] Q. Bai, K. D. Wise, and D. J. Anderson, "A high-yield microassembly structure for three-dimensional microelectrode arrays," IEEE Trans. Biomed Eng., vol. 47, no. 3, pp. 281-289, March 2000.

[2] E. M. Maynard, C. T. Nordhausen, and R. Normann, "The utah intracortical electrode array: a recording structure for potential brain-computer interfaces," Electroencephalography and clinical Neurophysiology, vol. 102, pp. 228-239, 1997.

[3] M. Nicolelis, "Actions from thoughts," Nature, vol. 409, pp. 403-407, 2001.

[4] R. R. Harrison, "A low-power integrated circuit for adaptive detection of action potentials in noisy signals," in Proc. of the 25-th Annual International Conference of the IEEE EMBS, September 2003, pp. 3325-3328.

[5] M. S. Lewicki, "A review of methods for spike sorting: the detection and classification of neural action potentials," Network: Computation in Neural Systems, vol. 9, no. 4, pp. R53-R78, 1998.

[6] Z. S. Zumsteg, R. E. Ahmed, G. Santhanam, K. V. Shenoy, and T. H. Meng, "Power feasibility of implantable digital spike-sorting circuits for neural prosthetic systems," in Proc. of the 26-th Annual International Conference of the IEEE EMBS, September 2004, pp. 4237-4240.

[7] D. A. Borkholder, "Cell based biosensors using microelectrodes," Ph.D. dissertation, Univ. of Stanford, Novermber 1998.

[8] K. Guillory and R. Normann, "A 100-channel system for real time detection and storage of extracellular spike waveforms," Journal of Neuroscience Methods, vol. 91, pp. 21-29, 1999.

[9] I. Obeid, J. C.Morizio, M. A. L. N. K. A. Moxon, and P. D. Wolf, "Two multichannel integrated circuits for neural recording and signal processing," IEEE Trans. on Biomed. Eng., vol. 50, no. 2, pp. 255-258, February 2003.

[10] A. Arieli, A. Sterkin, A. Grinvald, and A. Aertsen, "Dynamics of ongoing activity: Explanation of the large variability in evoked cortical responses,' Science, vol. 273, pp. 1868-1871, September 1996. 
[11] J. P. DONOGHUE, J. N. SANES, N. G. HATSOPOUlOS, and G. GAAL, "Neural discharge and local field potential oscillations in primate motor cortex during voluntary movements," The Journal of Neurophysiology, vol. 79, no. 1, pp. 159-173, January 1998.

[12] R. R. Harrison and C. Charles, "A low-power low-noise cmos amplifier for neural recording applications," IEEE Journal of Solid State Circuits, vol. 38, no. 6, pp. 958-965, June 2003.

[13] W. R. Patterson, Y. K. Song, C. W. Bull, I. Ozden, A. P. Deangellis, C. Lay, J. L. McKay, A. V. Nurmikko, J. D. Donoghue, and B. W. Connors, "A microelectrode/microelectronic hybrid device for brain implantable neuroprosthesis applications," IEEE Trans. on Biomed. Eng., vol. 51, no. 10, October 2004.

[14] P. Mohseni and K. Najafi, "A fully integrated neural recording amplifier with dc input stabilization," IEEE Trans. on Biomed. Eng., vol. 51, no. 5, May 2001.

[15] R. Lemon, Methods For Neuronal Recording In Conscious Animals. International Brain Research Organization, 1984.

[16] T. L. Deliyannis, Y. Sun, and J. K. Fidler, Continuous-Time Active Filter Design. CRC Press, 1999. 